\title{
Design, construction, and testing of lightweight $x$-ray mirror modules
}

\author{
Ryan S. McClelland*a, Michael P. Biskach ${ }^{\mathrm{a}}$, Kai-Wing Chan ${ }^{\mathrm{b}}$, Rebecca A. Espina ${ }^{\mathrm{a}}$, Bruce R. Hohl ${ }^{\mathrm{a}}$, \\ Elizabeth A. Matson ${ }^{c}$, Timo T. Saha ${ }^{c}$, William W. Zhang ${ }^{c}$ \\ ${ }^{a}$ SGT Inc. 7701 Greenbelt Road, Suite 400, Greenbelt, Maryland 20770, USA \\ ${ }^{\mathrm{b}}$ Center for Research and Exploration in Space Science and Technology, \\ University of Maryland, Baltimore County, Baltimore MD 21250, USA \\ ${ }^{\mathrm{c}}$ NASA Goddard Space Flight Center (GSFC), Greenbelt, MD USA 20771, USA
}

\begin{abstract}
Lightweight and high resolution optics are needed for future space-based X-ray telescopes to achieve advances in highenergy astrophysics. The Next Generation X-ray Optics (NGXO) team at NASA GSFC is nearing mission readiness for a 10 arc-second Half Power Diameter (HPD) slumped glass mirror technology while laying the groundwork for a future 1-2 arc-second technology based on polished silicon mirrors. Technology Development Modules (TDMs) have been designed, fabricated, integrated with mirrors segments, and extensively tested to demonstrate technology readiness. Tests include X-ray performance, thermal vacuum, acoustic load, and random vibration. The thermal vacuum and acoustic load environments have proven relatively benign, while the random vibration environment has proven challenging due to large input amplification at frequencies above $500 \mathrm{~Hz}$. Epoxy selection, surface preparation, and larger bond area have increased bond strength while vibration isolation has decreased vibration amplification allowing for space launch requirements to be met in the near term.
\end{abstract}

The next generation of TDMs, which demonstrates a lightweight structure supporting more mirror segments, is currently being fabricated. Analysis predicts superior performance characteristics due to the use of E-60 Beryllium-Oxide Metal Matrix Composite material, with only a modest cost increase. These TDMs will be larger, lighter, stiffer, and stronger than the current generation.

Preliminary steps are being taken to enable mounting and testing of 1-2 arc-second mirror segments expected to be available in the future. A Vertical X-ray Test Facility (VXTF) will minimize module gravity distortion and allow for less constrained mirror mounts, such as fully kinematic mounts. Permanent kinematic mounting into a modified TDM has been demonstrated to achieve 2 arc-second level distortion free alignment.

Keywords: slumped glass mirrors, module, Flight Mirror Assembly, FMA, X-Ray Optics, kinematic mount, VXTF

\section{INTRODUCTION}

Advancements in X-ray optics fabrication technologies are required to enable future discoveries by space-based X-ray telescopes [1]. While both lightweight and high resolution mirror fabrication technologies exist, no mature technology currently achieves both at once. For example, the Chandra mirror has a high resolution (0.5 arc-second HPD), but is relatively heavy $\left(18,000 \mathrm{~kg} / \mathrm{m}^{2}\right.$ collection area at $\left.1.0 \mathrm{keV}\right)$, while the Suzaku mirror has a low resolution (110 arc-second HPD), but is very lightweight (400 kg/m $\mathrm{m}^{2}$ at $\left.1.0 \mathrm{keV}\right)$ [2]. Technology currently in development by the NGXO team at NASA GSFC achieves both high angular resolution (10 arc-second HPD or better) and lightweight ( $400 \mathrm{~kg} / \mathrm{m}^{2}$ at 1.0 $\mathrm{keV}$ ) mirrors at a cost consistent with future NASA mission budgets. This slumped glass mirror technology is scalable to a variety of X-ray mission sizes and science objectives and has already been demonstrated to achieve 10 arc-second HPD performances in a full aperture X-ray test of multiple co-aligned mirror shells in a Technology Development Module (TDM) [2]. TDMs populated with co-aligned mirrors have been X-ray tested to verify performance, subjected to simulated space-flight environments, and then re-tested to ensure performance has not degraded. We seek to mitigate the cost and schedule risks of near term mission implementation of this optics technology through rigorous and repeated integration and testing of TDMs with increasing flight fidelity.

*ryan.s.mcclelland@nasa.gov; phone 1301 286-8615 
As the 10 arc-second technology nears mission readiness, the NGXO team is maturing a 1-2 arc-second technology based on polished silicon mirrors. In addition to the silicon polishing itself, efforts toward higher resolution include development of a Vertical X-Ray Test Facility (VXTF) and demonstration of a fully kinematic permanent mount. The VXTF will mitigate the gravity distortion imposed on the thin mirror segments during X-ray testing while the kinematic permanent mount will eliminate mirror distortion caused by bonding.

\subsection{Slumped Glass Mirror Module Technology Overview}

Fabrication of a slumped glass mirror module starts with $0.4 \mathrm{~mm}$ thick glass sheets slumped over polished convex mandrels [3]. The mirror segments thus formed are cut to size (200 mm axial length and variable azimuthal span), coated with a thin layer of iridium, and temporarily mounted to a hexapod manipulator allowing for rigid body alignment. Pairs of mirrors are then aligned to a common focus, permanently bonded into the module structure, and finally released from the temporary mount. Once the module is fully populated with mirror segments, the back of the module is closed out with a protective panel and the assembly is tested. Many modules are then co-aligned into a common structure to complete the Flight Mirror Assembly (FMA) as shown in Figure 1.

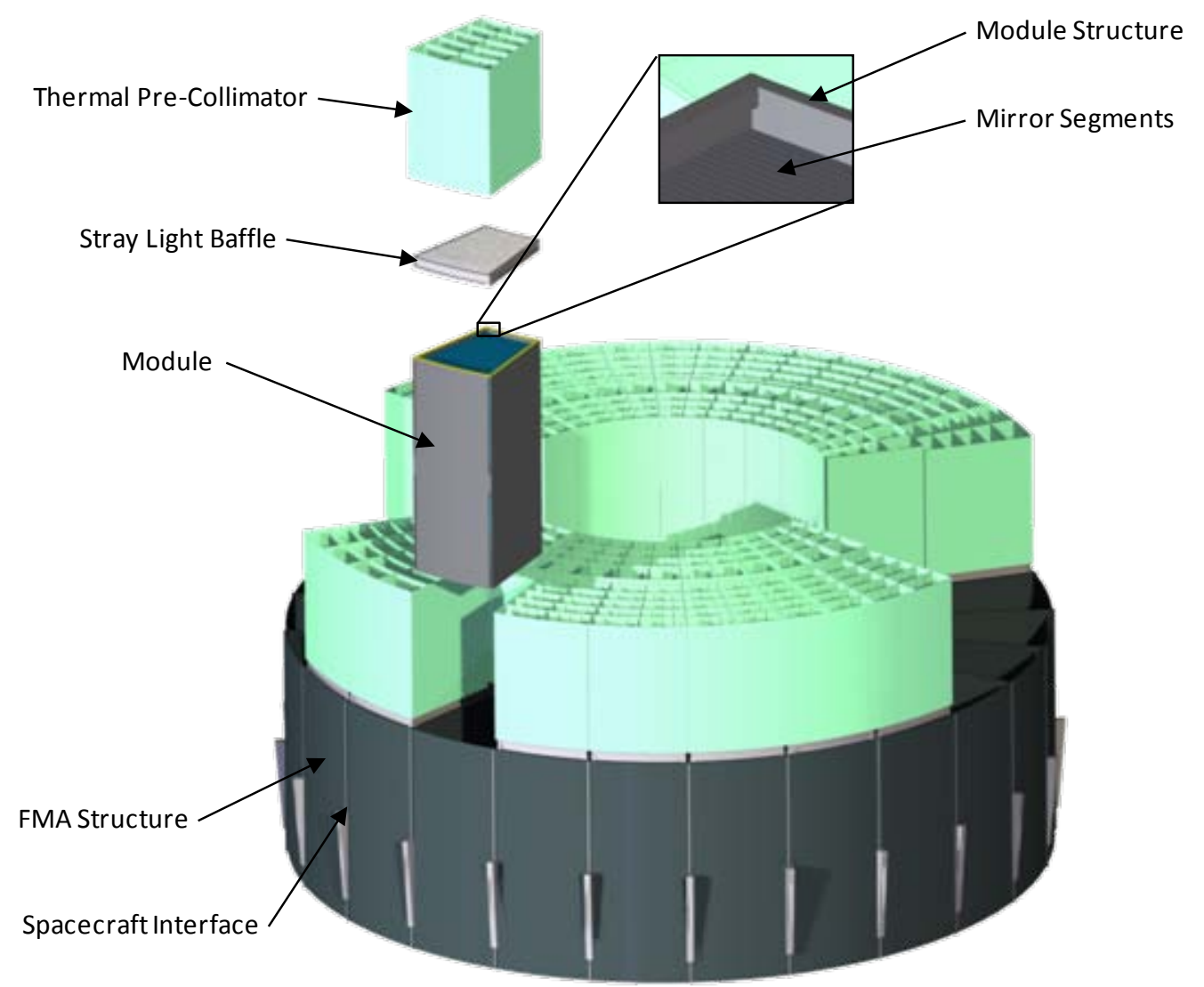

Figure 1. Exploded view of an FMA with two partially populated rings of modules.

\subsection{Flight Mirror Assembly Overview}

The FMA combines tens of modules collectively containing thousands of mirror segments into a common structure with a common focus (though some missions studied have multiple foci). Significant FMA design and analysis was performed as part of the International X-Ray Observatory (IXO) mission study [4]. The design approach of combining rings of modules into a Carbon Fiber Reinforced Polymer (CFRP) super structure is scalable to a variety of mission sizes including Flagship Missions such as IXO, Missions of Opportunity, and Explorer Missions. The effective area at specific energies of an FMA design can be adjusted by changing the number and diameter of module rings. 


\section{PROGRESS WITH TECHNOLOGY DEVELOPMENT MODULES}

The NGXO team has achieved notable progress with the current generation of TDMs, which hold three co-aligned primary and secondary mirror pairs, including demonstration of 11 arc-second full aperture X-ray resolution and survival of simulated space-flight environments without performance degradation. Progress has been achieved through repeated construction of TDMs populated with co-aligned mirrors as well as repeated environmental testing. The process of permanently bonding the mirrors to the TDM structure has been tuned to achieve minimal bonding distortion and sufficient bond strength. TDMs have undergone random vibration, acoustic, and thermal vacuum tests without breaking or plastically deforming the bonds, as demonstrated by post environmental X-ray performance testing. The random vibration environment has proven particularly challenging.

\subsection{Technology Development Module Design}

The current generation of TDMs were designed to hold three pairs of co-aligned primary and secondary mirrors (6 mirror segments total) in a low cost, rigid, CTE matched structure. Figure 2 illustrates the design. To keep costs low and eliminate issues associated with structure self-weight deformation the structure consists of several relatively thick solid metal panels fastened together. Kovar F15 was selected as the structural material due to the relatively good CTE match with the D263 mirror glass (6.7 ppm/C vs. $6.3 \mathrm{ppm} / \mathrm{C}$ ), and relatively low raw materials and fabrication costs [5]. The TDM is kinematically supported during integration, $\mathrm{X}$-ray testing, and environmental testing using three spherical tooling balls in three grooves or bushings. To minimize self-weight distortion, there are separate sets of kinematic mounts used for vertical and horizontal performance testing.
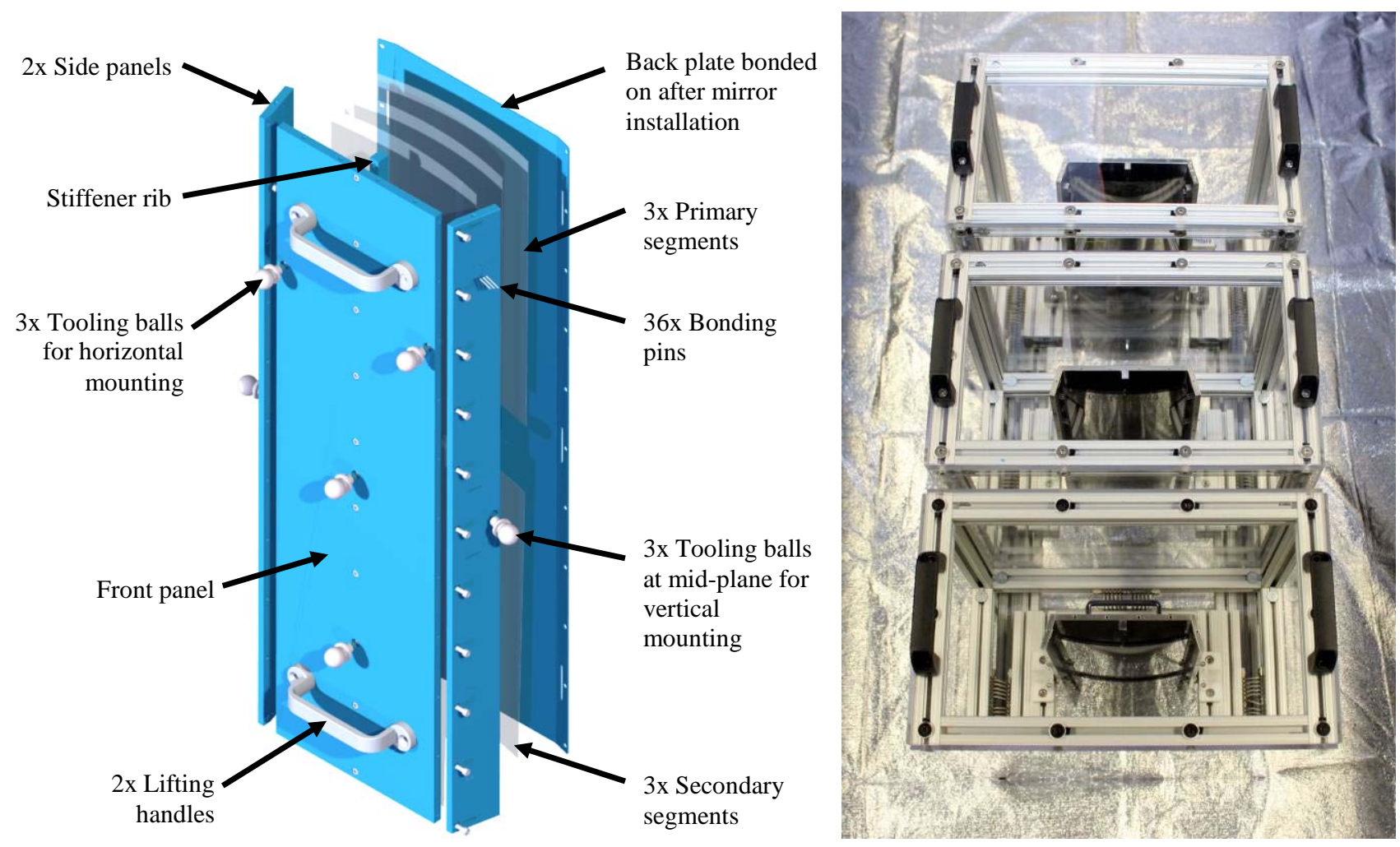

Figure 2. Exploded view of a TDM (left) and top view of three fully populated TDMs in shipping/displace cases (right).

The TDM structure was also designed to undergo environmental testing to generic space-flight mission requirements [5]. An optional back plate can be attached to close-out the structure for additional stiffness, foreign object protection, and acoustic attenuation. Bonding the back plate onto the TDM has been demonstrated without optical performance degradation. Fastening the back plate with screws degraded the optical performance by $>60$ arc-second. 


\subsection{Technology Development Module Bonding}

The TDM structures are integrated with co-aligned mirror segments inside the Vertical Alignment Facility (VAF) shown in Figure 3. Alignment and bonding of six mirror segments has been demonstrated many times and can currently be accomplished in one week. Due to the thermal sensitivity of the TDMs, the VAF is kept at $20^{\circ} \mathrm{C} \pm 0.05^{\circ} \mathrm{C}$ using continuous horizontal flow of precision temperature air across the alignment facility. Temperature stability as good as $20^{\circ} \mathrm{C} \pm 0.005^{\circ} \mathrm{C}$ has been demonstrated over a 24 hour period.
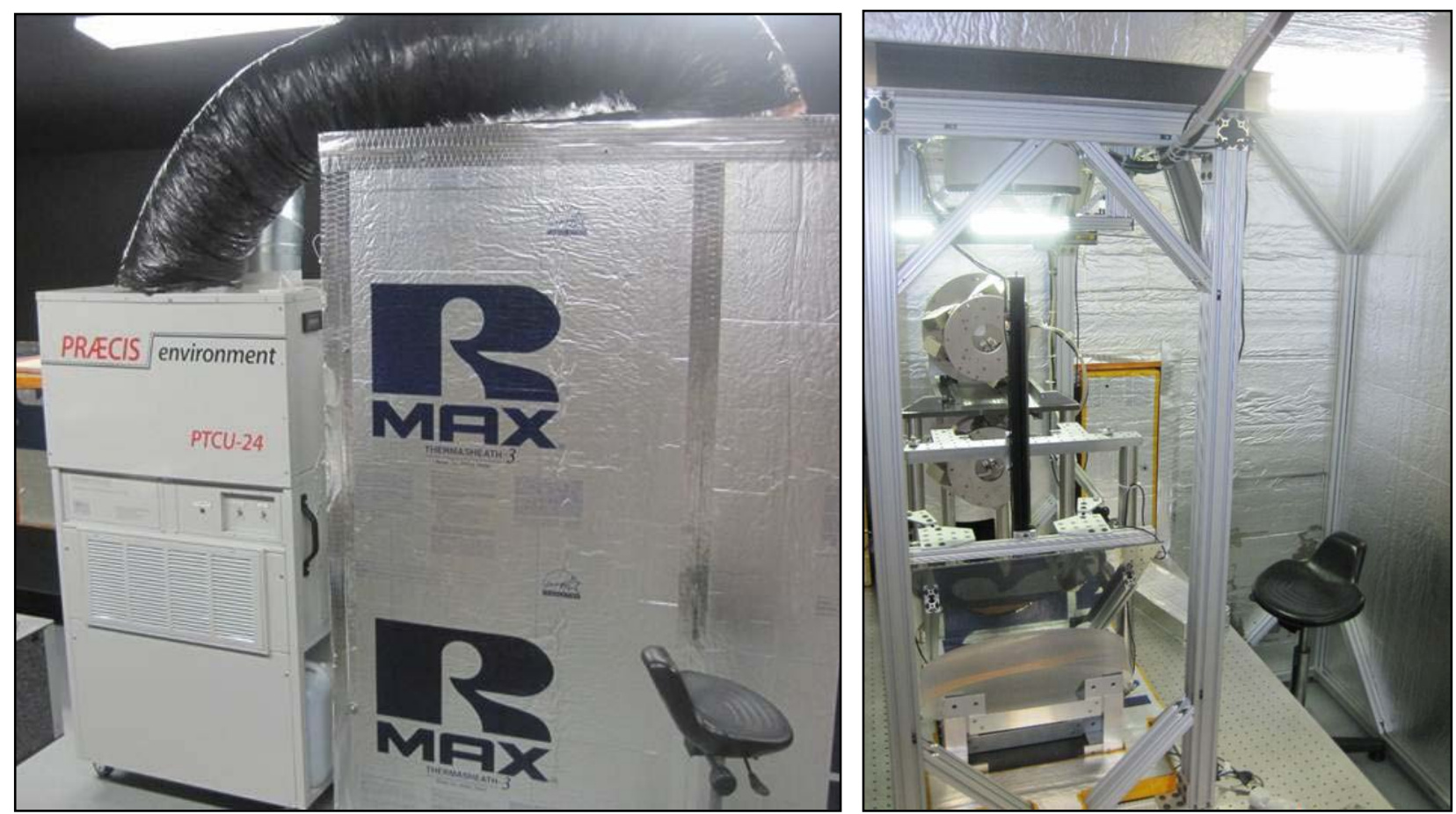

Figure 3. Outside view of the Vertical Alignment Facility (VAF) thermal room and precision temperature air generator (left) and inside view of the VAF (right).

During TDM integration, each mirror is temporarily mounted to a hexapod manipulator by a three sphere Kelvin Clamp kinematic mount for distortion free rigid body alignment. Once aligned, the mirror is bonded at six locations along the axial edges and permanently attached to the TDM structure. There are three bonds attaching the mirror segments to the TDM, as shown in Figure 4. The P0 bond, applied and cured before the mirror is integrated, attaches a metal clip to the glass mirror segment in order to prevent stress concentrations and provide a larger area for the P1 bond. The P1 bond attaches the clip to a pin which slides within a close-fit hole in the TDM structure. Finally, the P2 bond secures the pin to the TDM structure.

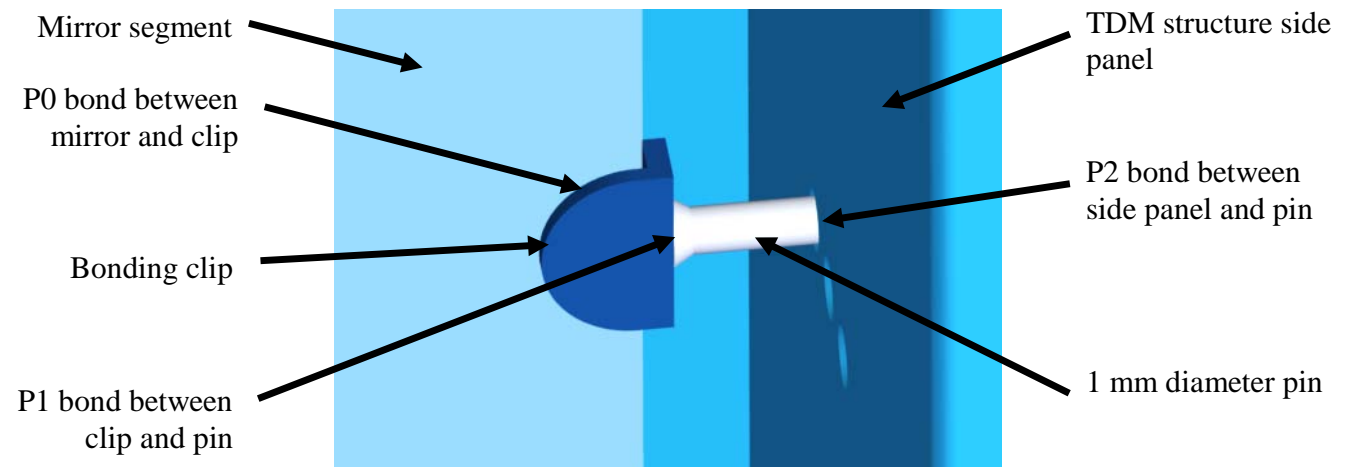

Figure 4. Detail of structural connection between mirror segments and TDM structure including P0, P1, and P2 bonds. 


\subsection{Technology Development Module Results}

Many fully integrated TDMs have undergone X-ray performance testing and several have undergone environmental testing as well. A single unit has undergone six X-ray tests, two thermal vacuum tests, two random vibration tests, and one acoustic test. Figure 3 shows three completed TDMs with 18 total integrated mirror segments.

\section{$X$-ray performance testing}

X-ray tests have been performed at GSFC's $600 \mathrm{~m}$ beam-line facility. The 8 inch diameter horizontal beam fully illuminates the mirror segments within the TDM. X-ray performance of 11-13 arc-second has been repeatedly achieved. If the effects of gravity are analytically removed from the X-ray image, the predicted performance is 9 arc-second. The $\mathrm{X}$-ray image correlates well with the predicted image based on the combination of normal incidence interferometric mirror metrology and Finite Element Analysis (FEA) self-weight distortion results as shown in Figure 4.

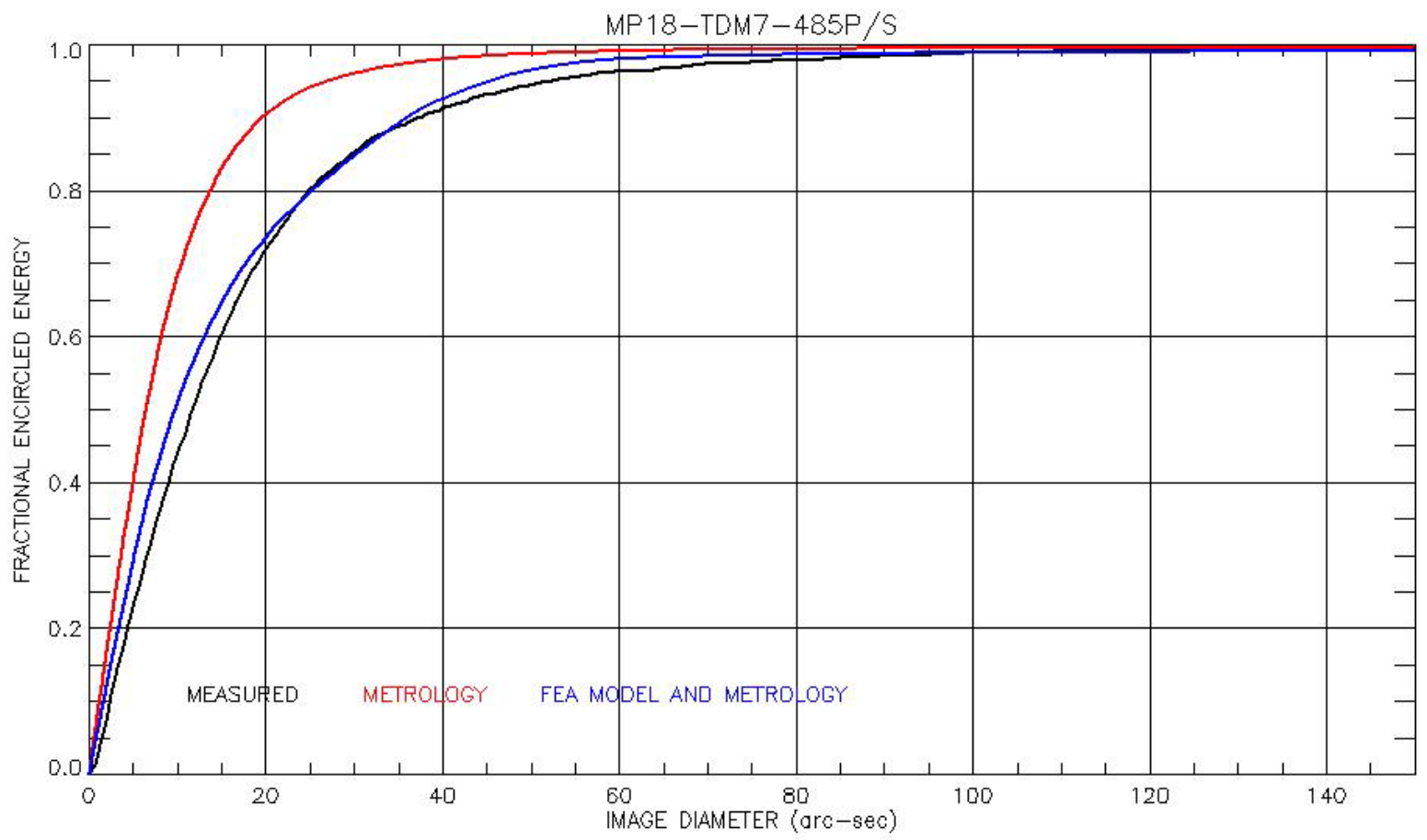

Figure 4. Encircled energy as measured during X-ray testing, predicted from normal incidence metrology, and predicted from a combination of normal incidence metrology and FEA modeling of gravity distortion. The effect of gravity is significant and well correlated with measurement.

\section{Acoustic testing}

A TDM was tested in NASA GSFC's Reverberant Chamber Acoustic Test Facility. The test article inside the chamber is shown in Figure 5. Stresses induced by acoustic loads are often a concern for thin and lightweight spacecraft structures, such as equipment panels and solar arrays, and there was a concern that the thin glass mirror segments would be damaged by acoustic loads. However, since the TDM structure is closed on four sides, the sound pressure waves are too large to act coherently on the mirrors and the resulting stress is low. Response acceleration, as measured by an accelerometer located on a mirror segment, was 7.6 Grms at the full level of $143.3 \mathrm{~dB}$ Overall Sound Pressure Level (OASPL). Compared with the response acceleration from vibration testing, which were as high as $44.8 \mathrm{Grms}$, acoustic loads are relatively inconsequential. Post-test inspection and X-ray testing verified acoustic loading did not damage the TDM nor degrade the optical performance. Based on these results, acoustic loads are not expected to be a driving requirement for the technology. The acoustic levels used for this test envelope those of likely launch vehicles for a future X-ray mission. 

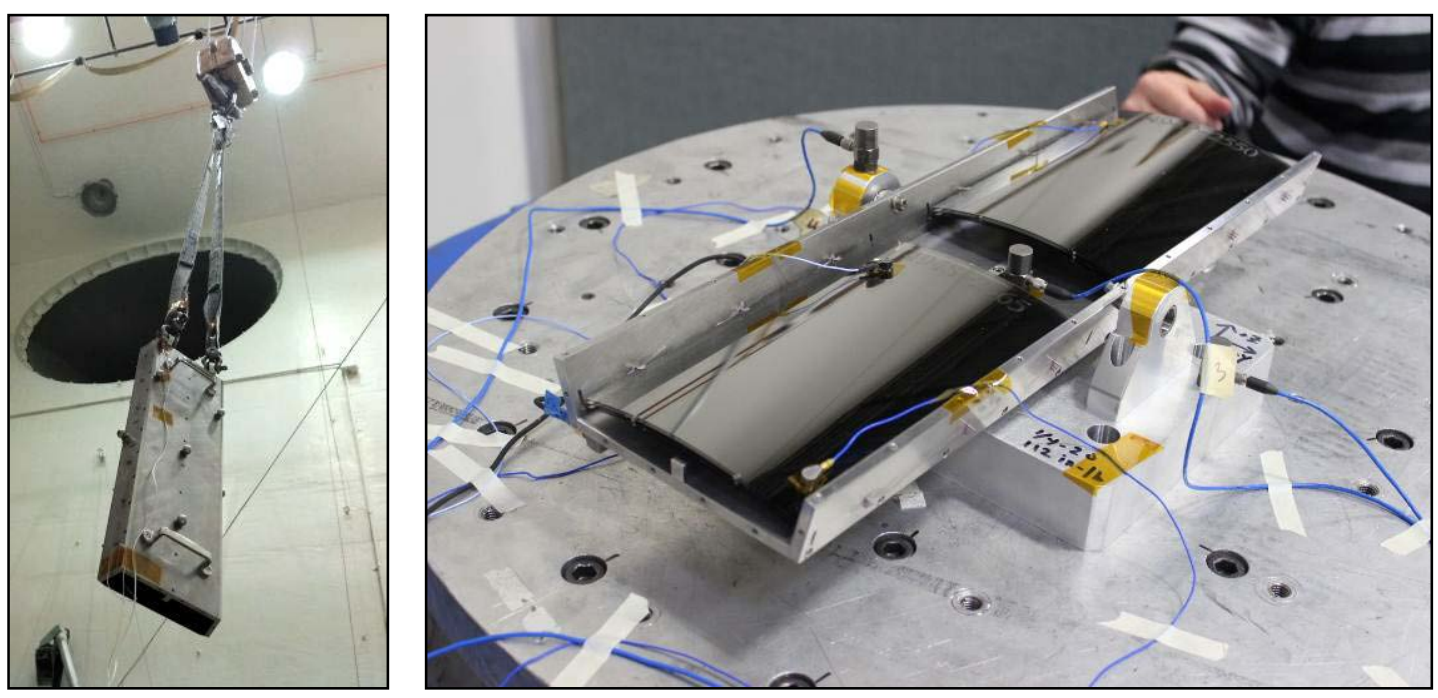

Figure 5. TDM in reverberant chamber acoustic load testing (left) and random vibration testing (right).

\section{Thermal vacuum testing}

The same TDM which underwent acoustic testing was thermal vacuum tested in two stages of successively larger temperature excursions. The goal was to verify the TDM could endure the survival temperature limits of the Chandra mirrors, $10^{\circ} \mathrm{C}$ to $30^{\circ} \mathrm{C}$, without degrading performance [5]. The TDM operates at $20.0^{\circ} \mathrm{C} \pm 0.1^{\circ} \mathrm{Ci}$ and is not expected to operate at the survival temperatures, which are defined for mission contingencies. The temperature profile used is shown in Figure 6. Post-test X-ray performance verified the thermal vacuum environment did not degrade the performance. A subsequent test from $0^{\circ} \mathrm{C}$ to $40^{\circ} \mathrm{C}$ was performed in an attempt to widen the survival temperature range for the TDM, which can simplify spacecraft thermal, power, and attitude control systems. Again, post-test X-ray performance verified the thermal vacuum environment did not degrade the performance, indicating that further expansion of the survival temperature range may be possible. Based on these results, the thermal vacuum environment is not expected to be a driving requirement for the technology, though future testing will define the temperature limits for mission design.

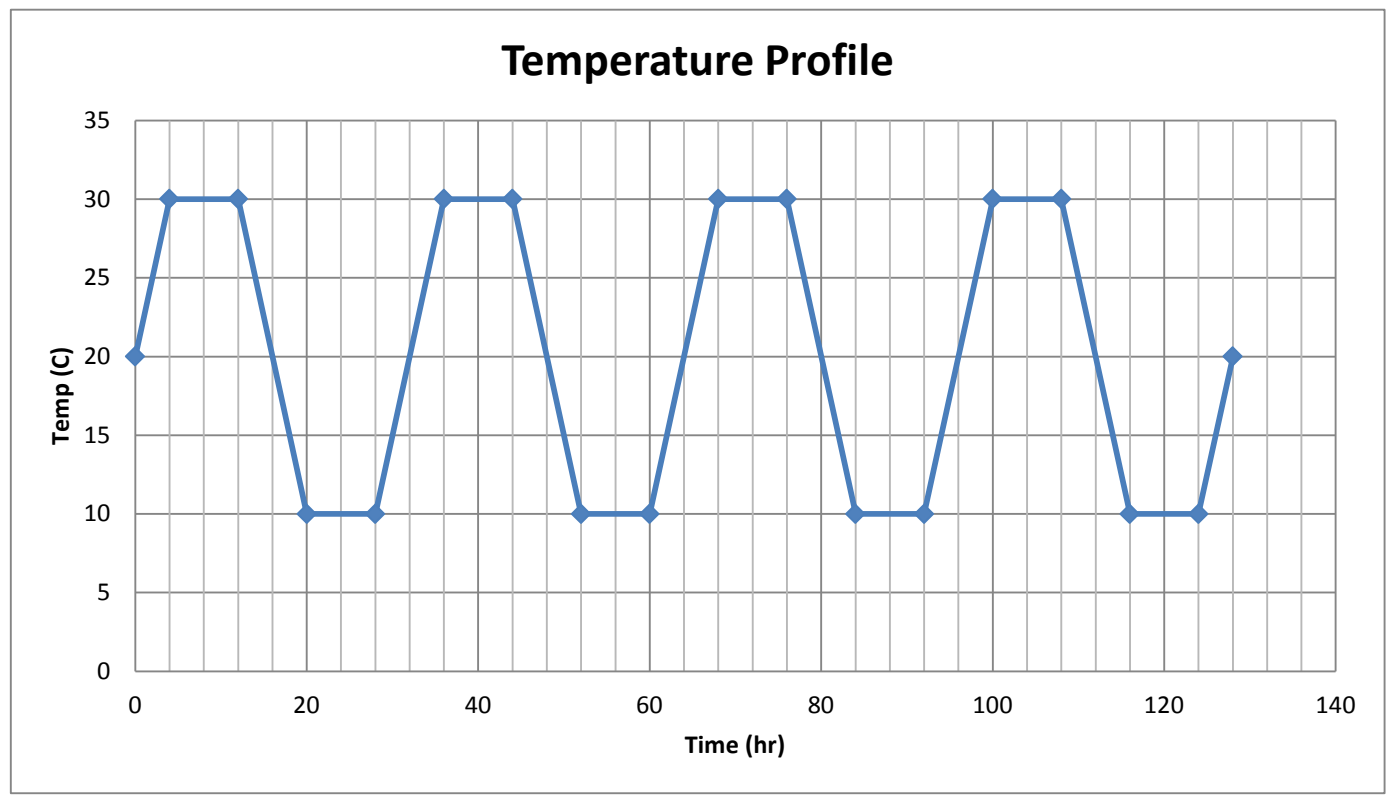

Figure 6. Temperature profile used for $10^{\circ} \mathrm{C}$ to $30^{\circ} \mathrm{C}$ thermal vacuum test of a TDM. 


\section{Random vibration development testing}

Four random vibration tests of increasing input levels have been performed on TDMs, including two on the same unit. Due to the difficulty of accurately predicting failure of the mirrors and bonded joints with FEA, an iterative approach to demonstrating the strength of the populated TDMs was adopted, whereby each test demonstrated increased strengthening of the platform. The goals of vibration testing were to characterize the dynamic response of the TDM to random vibration input, expected to be the driving load case for launching the optics, and demonstrate survival without performance degradation up to the NASA GSFC generic requirement of 6.8 Grms [5].

The first test was performed with the goal of characterizing the dynamic response behavior of the TDM while inputting significant accelerations to demonstrate the strength of the test unit. Five accelerometers were attached to the test setup as shown in Figure 5, including two miniature one-axis accelerometers attached directly to the mirror segments, to record random vibration response spectra. This instrumentation setup was preserved throughout the sequence of four vibration tests to allow direct response comparison between each test. The maximum input acceleration, average mirror response, and resulting amplification factor for each test and each axis are shown in Table 1. There are large mirror responses above $500 \mathrm{~Hz}$ that were not predicted by the FEA model, resulting in large amplification of the input accelerations, as shown in Figure 7.

During the first vibration test, mirror breakage was expected to be the mode of failure. No failure was noted during posttest mirror inspection. However, once the TDM was optically tested, the performance had significantly degraded. Closer inspection revealed about one-third of the 36 P1 bonds had failed. For the next test, a stronger epoxy was selected and coupon tested to verify the increased strength. Coupon testing showed that Hysol 9309NA increased the bond strength by a factor of 2.6 over the JB-Weld 8265 used during the first vibration test as shown in Table 2 .

Table 1. Summary of vibration test inputs and resulting mirror acceleration.

\begin{tabular}{|c|c|c|c|c|c|c|c|c|c|c|c|c|}
\hline \multirow[b]{2}{*}{$\begin{array}{c}\text { Test } \\
\text { Axis }\end{array}$} & \multicolumn{3}{|c|}{ Test 1} & \multicolumn{3}{|c|}{ Test 2} & \multicolumn{3}{|c|}{ Test 3} & \multicolumn{3}{|c|}{ Test 4} \\
\hline & $\begin{array}{c}\text { Input } \\
\text { Accel. } \\
\text { (Grms) }\end{array}$ & $\begin{array}{c}\text { Mirror } \\
\text { Accel. } \\
\text { (Grms) }\end{array}$ & $\begin{array}{l}\text { Amplif- } \\
\text { ication } \\
\text { Factor }\end{array}$ & $\begin{array}{c}\text { Input } \\
\text { Accel. } \\
\text { (Grms) }\end{array}$ & $\begin{array}{c}\text { Mirror } \\
\text { Accel. } \\
\text { (Grms) }\end{array}$ & $\begin{array}{l}\text { Amplif- } \\
\text { ication } \\
\text { Factor }\end{array}$ & $\begin{array}{c}\text { Input } \\
\text { Accel. } \\
\text { (Grms) }\end{array}$ & $\begin{array}{c}\text { Mirror } \\
\text { Accel. } \\
\text { (Grms) }\end{array}$ & $\begin{array}{l}\text { Amplif- } \\
\text { ication } \\
\text { Factor }\end{array}$ & $\begin{array}{c}\text { Input } \\
\text { Accel. } \\
\text { (Grms) }\end{array}$ & $\begin{array}{c}\text { Mirror } \\
\text { Accel. } \\
\text { (Grms) }\end{array}$ & $\begin{array}{l}\text { Amplif- } \\
\text { ication } \\
\text { Factor }\end{array}$ \\
\hline $\mathrm{X}$ & 2 & 16.9 & 8.5 & 2.8 & 20.55 & 7.3 & 3.6 & 39.2 & 10.9 & 6.4 & 26.8 & 4.2 \\
\hline $\mathrm{Y}$ & 2.4 & 16.35 & 6.8 & 3 & 18.8 & 6.3 & NA & NA & NA & 4.3 & 13.3 & 3.1 \\
\hline $\mathrm{Z}$ & 2.8 & 15.9 & 5.7 & 2.8 & 9.55 & 3.4 & NA & NA & NA & 6.8 & 9.8 & 1.4 \\
\hline Result & \multicolumn{3}{|c|}{12 of 36 bonds failed } & \multicolumn{3}{|c|}{ No damage or degradation } & \multicolumn{3}{|c|}{ Intentional test to failure } & \multicolumn{3}{|c|}{ No damage some degradation } \\
\hline
\end{tabular}




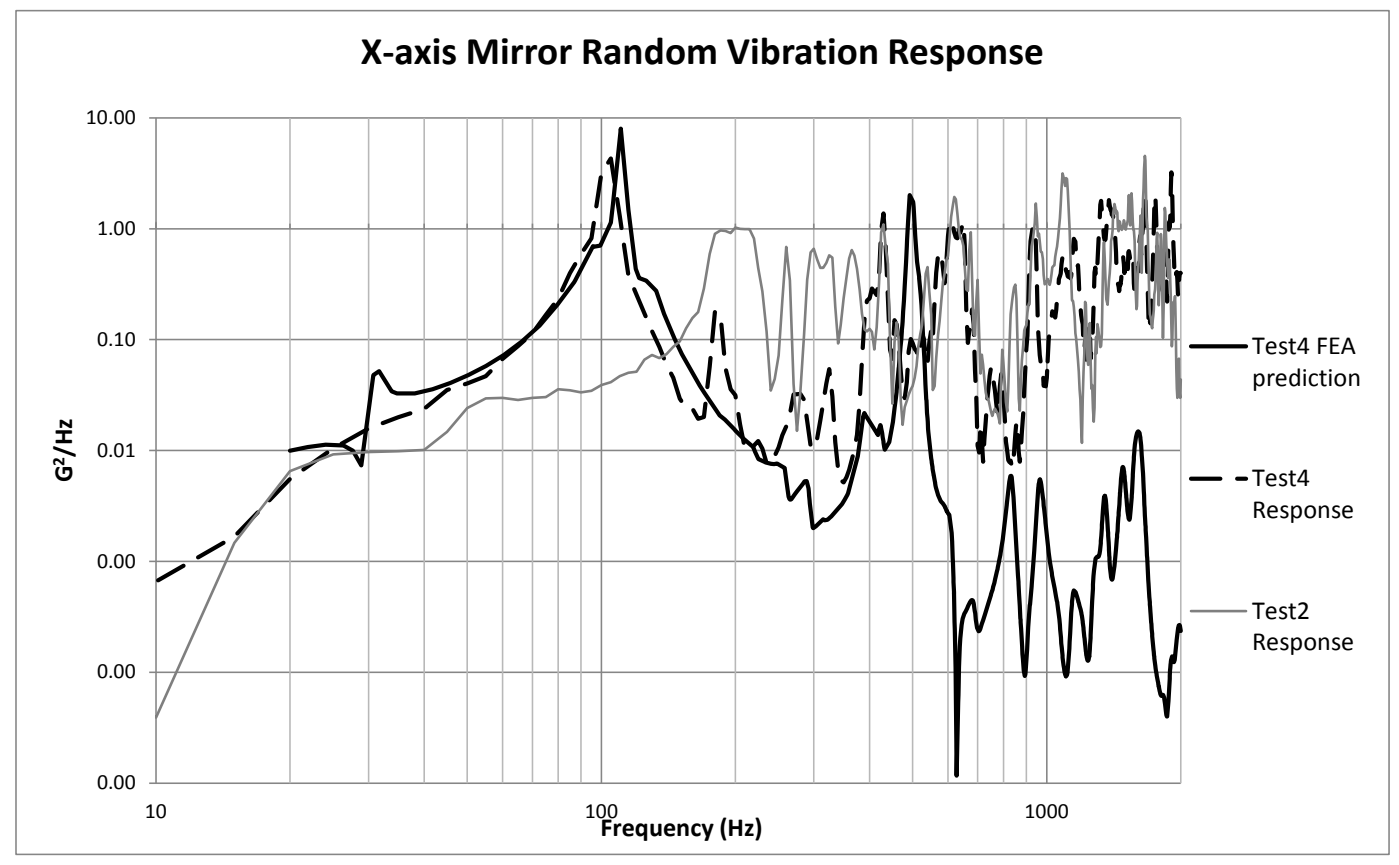

Figure 7. Mirror response accelerations Power Spectral Density (PSD) plot showing higher than expected response above $500 \mathrm{~Hz}$.

Table 2. Coupon testing results showing increased strength with epoxy selection and bond diameter/area increase.

\begin{tabular}{|c|c|c|}
\hline P1 bond & $\begin{array}{c}\text { Bending strength } \\
\left(\mathbf{N}^{*} \mathbf{m m}\right)\end{array}$ & Std. Dev. (N*mm) \\
\hline JB-Weld 8265 w/ 1.5 mm bond $ø$ & 12.9 & 2.2 \\
\hline Hysol 9309NA w/ 1.5 mm bond $ø$ & 33.8 & 3.1 \\
\hline Hysol 9309NA w/ 2.0 mm bond $ø$ & $\mathbf{8 0 . 1}$ & 9.3 \\
\hline
\end{tabular}

The second random vibration test was performed with the goal of demonstrating the stronger Hysol 9309NA epoxy P1 bonds could survive input vibration levels higher than the previous test without breaking or measurably distorting the optics. The test completed successfully without any visible damage to the mirrors or bonds. Post vibration X-ray testing verified the performance had not changed as a result of random vibration testing. Based on the high root mean square acceleration (Grms) seen by the mirror, the mirrors likely experienced transient accelerations above $75 \mathrm{~g}$.

The third random vibration test was designed to explore the limits of the TDM's strength by intentionally testing until failure. This test was performed only in the $\mathrm{X}$ axis, since the amplification factors were generally highest in that axis. After each test level, the bonds and mirrors were checked for failure before going on to the next higher input vibration level. The P1 bonds began to fail at 3.6 Grms input, below the 6.8 Grms requirement, indicating additional strengthening was required.

During the first three vibration tests, the TDM was rigidly mounted to the shaker table with an extremely stiff fixture having a first mode $>2,000 \mathrm{~Hz}$. It was hypothesized that a less rigid fixture, similar in frequency to a flight-like mount would help filter out the high frequency mirror response. To test this hypothesis, the vibration fixture shown in Figure 8, having a first mode of $120 \mathrm{~Hz}$, was designed, analyzed, and fabricated.

The fourth random vibration test explored the effect of the vibration isolation fixture on the dynamic response of the TDM. As expected, the response accelerations of the mirror declined dramatically, reducing the input amplification by about a factor of two as shown in Table 1. The TDM was tested at or near the required $6.8 \mathrm{Grms}$ input level, except in the Y-axis where conservatism was required to ensure all three axes were tested. Post-test inspection did not reveal any damage to the mirror or bonds, though subsequent X-ray testing revealed one mirror pair had significantly degraded. This result likely indicates one or more bonds plastically deformed during vibration testing. 


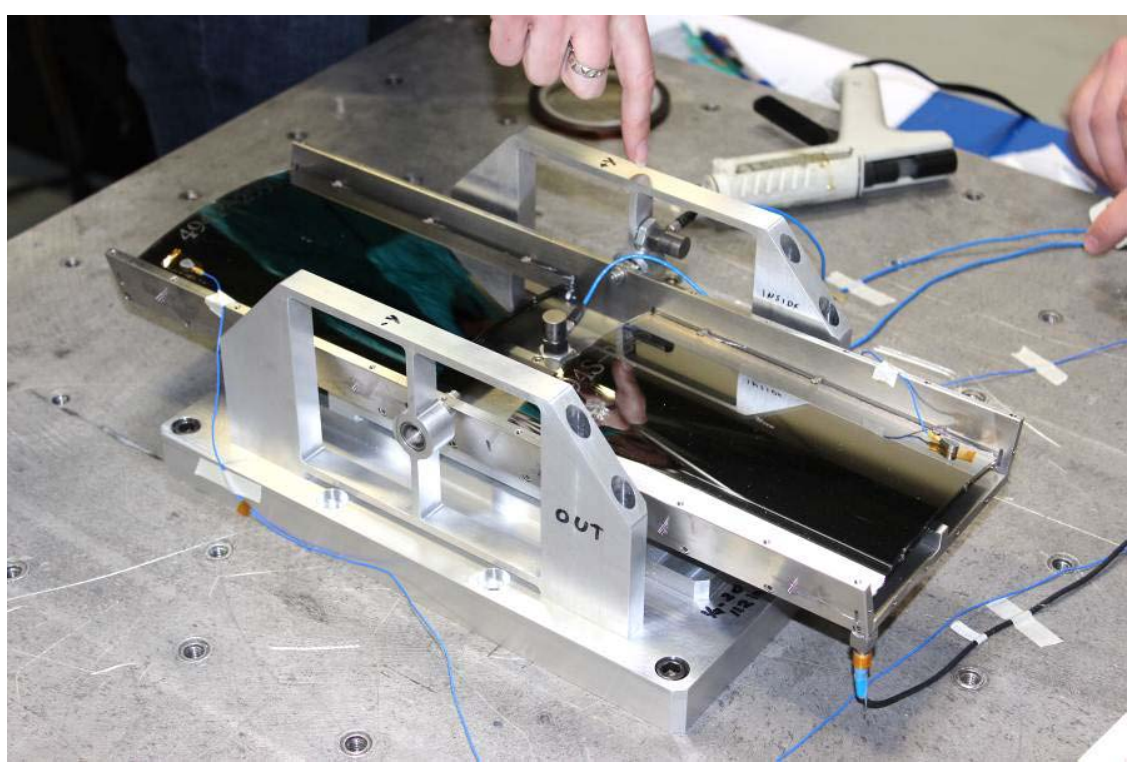

Figure 8. TDM with vibration isolation fixture on the shaker table.

To further strengthen the P1 bonds, a larger bond area was adopted. Coupon testing indicates the larger area provides about two times greater bond strength as shown in Table 2. With a combination of the vibration isolation fixture and doubled bond strength the TDM is expected to surpass the required random vibration levels. This will be verified by future testing. NGXO expects to receive Technology Readiness Level 5 (TRL 5) certification subsequent to a successful test.

\section{FUTURE TECHNOLOGY DEVELOPMENT MODULES}

The current TDM platform has been used to demonstrate co-alignment of multiple mirror paris, X-ray performance below 10 arc-second, and compatibility with space-flight environments. The subsequent generation of TDMs will improve upon this by mounting a larger range of mirror segment sizes and demonstrating a lightweight structure.

\subsection{Design of next generation Technology Development Modules}

The next generation of TDM, shown in Figure 9 and currently in fabrication, is intended to bridge the gap between the current breadboard platform and a future module designed to be space-flight qualified for a 10 arc-second telescope Xray mission. If fully populated with mirror segments, the next generation TDM would hold $\sim 100$ mirrors with a total mass of $1.7 \mathrm{~kg}$. The structure itself weighs only $1.1 \mathrm{~kg}$, demonstrating a structure/mirror mass ration of 0.65 . The ability to create a low mass structure with high stiffness is enabled by switching from the Kovar F15 material used in the current TDMs to Materion E-60, a Beryllium Oxide Metal Matrix Composite (MMC) with a stiffness to weight ratio more than seven times that of Kovar. The CTE of E-60 is also a nearly perfect match to the D263 mirror glass. However, the E-60 material cannot be milled and tapped using conventional machining; it must be fabricated with Electrical Discharge Machining (EDM) at a facility which can accommodate the hazardous byproducts of Beryllium. The next generation design, with solid E-60 walls, strikes a balance between structural simplicity for cost reduction and light weight. Additional weight savings can be realized in future module designs by using isogrid panel structures, though at additional fabrication cost. 


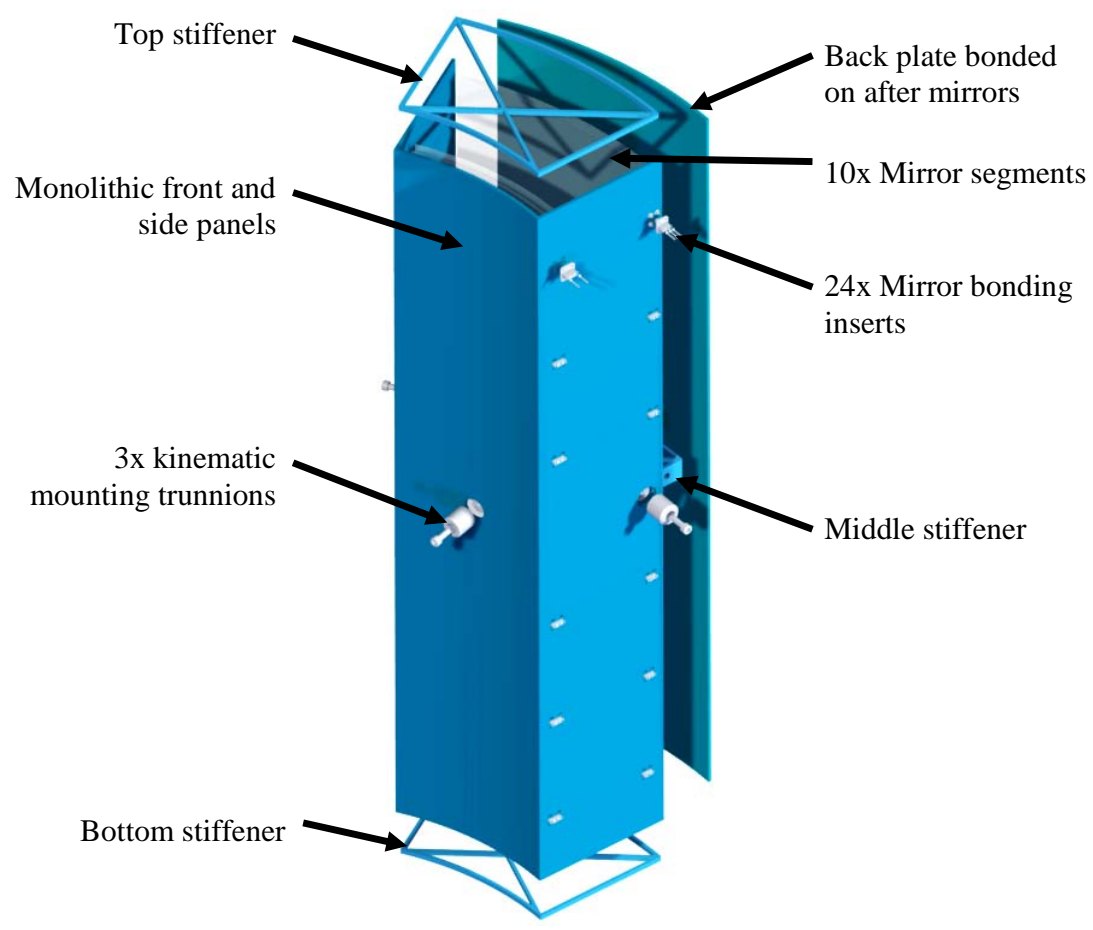

Figure 9. Exploded view of the next generation TDM, currently in fabrication. The structure is bonded together from E-60 Beryllium Oxide composite components.

\subsection{Analysis of next generation Technology Development Modules}

In addition to being larger and lighter than the current TDMs, the next generation will also be significantly stiffer which reduces self-weight distortion, low frequency vibration amplification, and mirror/bond stress. Figure 10 illustrates the $849 \mathrm{~Hz}$ first mode of the E-60 TDM structure. The mode falls to $319 \mathrm{~Hz}$ when a Kovar structure is assumed. Figure 10 also shows the expected distortion due to gravity in the horizontal X-ray test configuration.
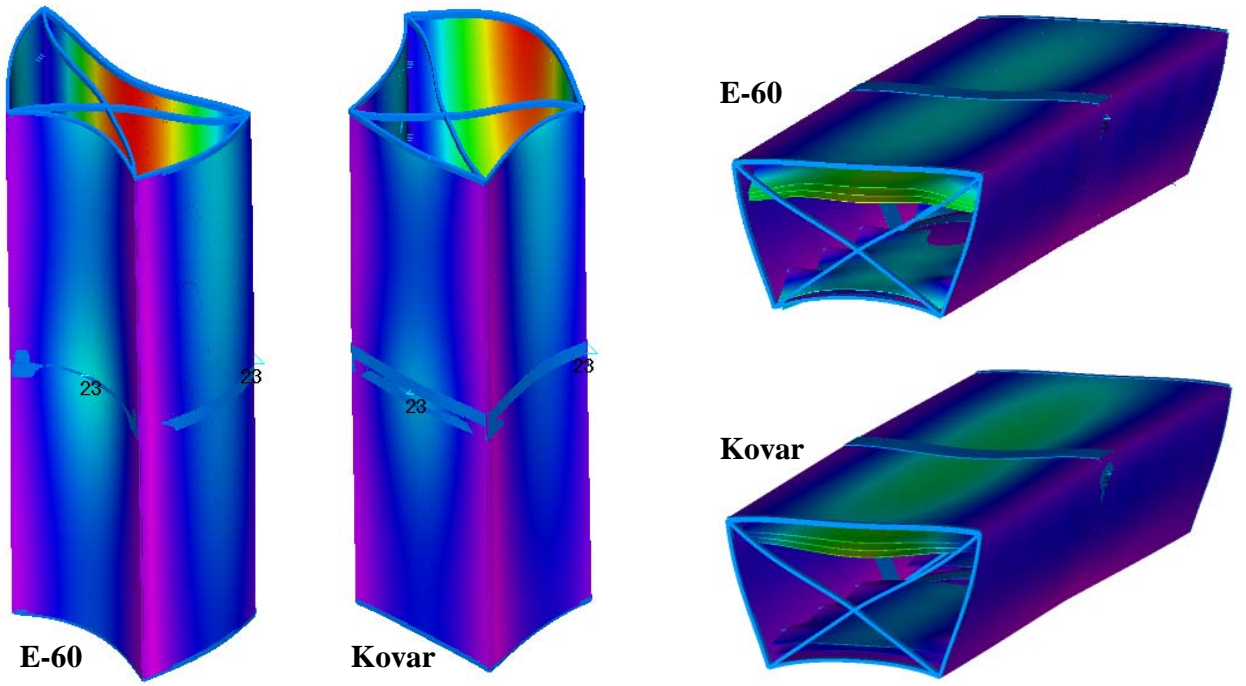

Figure 10. First mode of next generation TDM structure is $849 \mathrm{~Hz}$ when fabricated from E-60 and $319 \mathrm{~Hz}$ when fabricated from Kovar (left). The gravity distortion figure error in the horizontal X-ray configuration is 2.2 arc-seconds HPD with an E-60 structure and 5.8 arc-seconds HPD with a Kovar structure (right). 


\section{MOUNTING AND TESTING OF 1 ARC-SECOND LEVEL X-RAY OPTICS}

To achieve our longer term goal of building X-ray optics with a resolution of 1-2 arc-second, different X-ray testing and mirror integration approaches will be necessary. A Vertical X-ray Test Facility (VXTF) is being developed that will minimize the effect of gravity distortion during X-ray testing, which is already becoming a performance driver for the 10 arc-second technology. The move to a vertical X-ray beam will allow for new approaches to mirror segment mounting, such as ideal kinematic constraint [5].

\subsection{Design of a Vertical X-Ray Test Facility}

A unique Vertical X-ray Test Facility is being planned that will use the existing $600 \mathrm{~m}$ beamline as a collimated X-ray source as shown in Figure 11. The collimated beam will be folded once by a 12" flat mirror with a multilayer coating capable of reflecting $0.1 \mathrm{eV}$ X-rays, focused by the TDM being tested, and imaged by a CCD at the bottom of the $12 \mathrm{~m}$ tube. Large diameter stainless steel tubes are readily and inexpensively available from the oil industry and installation services are readily and inexpensively available from the industrial construction industry. The X-ray source and CCD are also readily available, resulting in a facility that can be created from off-the-shelf technologies and services.

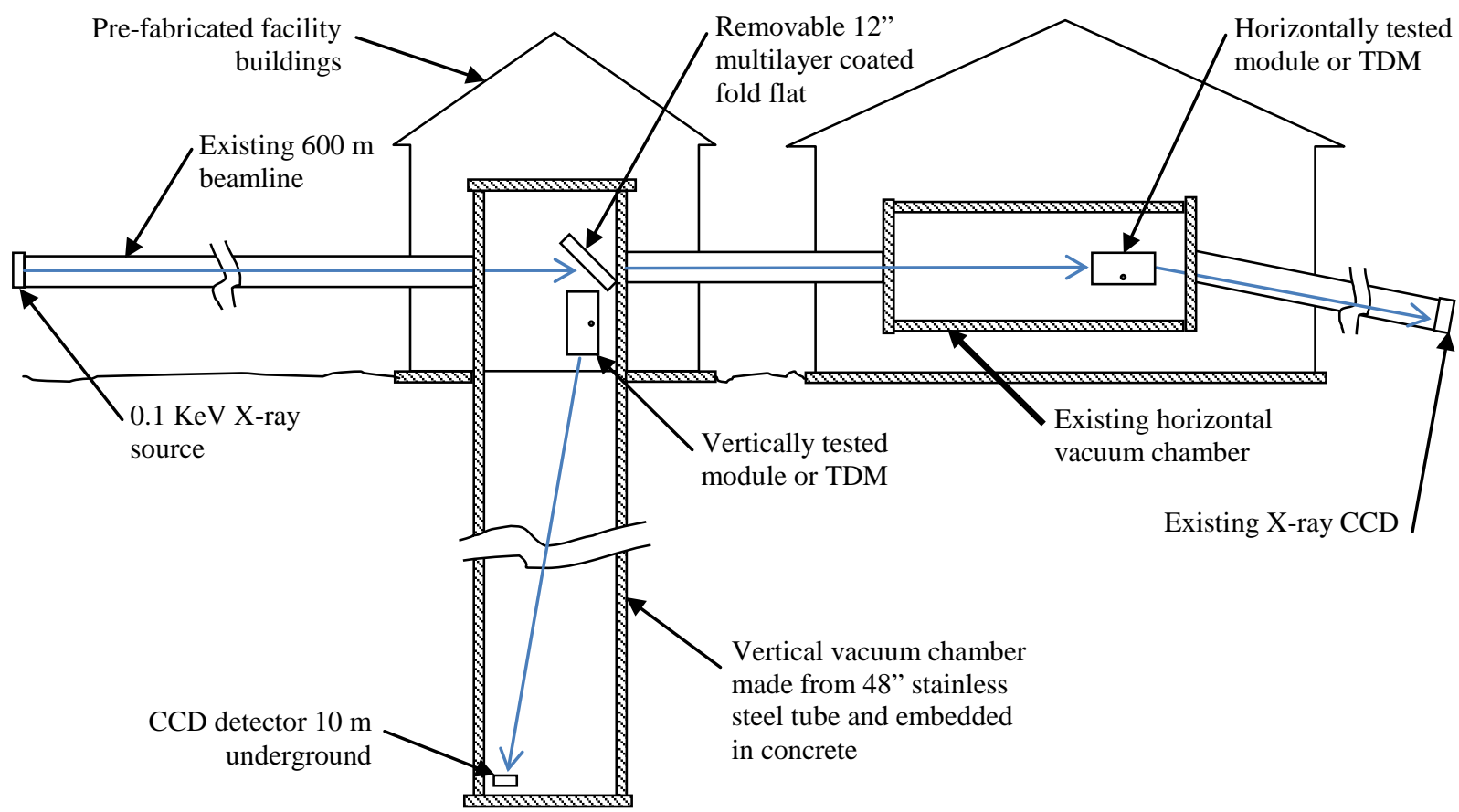

Figure 11. Layout of planned Vertical X-Ray Test Facility (VXTF) which preserves the capabilities of the existing $600 \mathrm{~m}$ beamline at NASA GSFC.

\subsection{Kinematic Permanent Mount}

Moving from the current mirror mounting technique, which constrains 36 degrees of freedom, to a kinematically constrained mirror mount eliminates or greatly reduces mounting induced distortion. In particular, the mirror segment is more than an order of magnitude less sensitive to bond distortions when kinematically constrained as compared to an over-constrained mirror [5]. The ability to align and mount a pair of kinematically constrained mirrors by transferring the kinematic mechanisms from an alignment fixture into a modified TDM, shown in Figure 12, has been demonstrated. A mirror pair was permanently bonded into the modified TDM without changing the alignment more than 1 arc-second and without mirror figure distortion. Using this method, the slumped glass mirrors can permanently mount at the limit of their fabrication, currently 5-6 arc-second. When better segments become available from Silicon polishing [2] these can be kinematically mounted and tested in the VXTF with minimal distortion. The next step is to vibration test the assembly to verify it can withstand launch loads. 


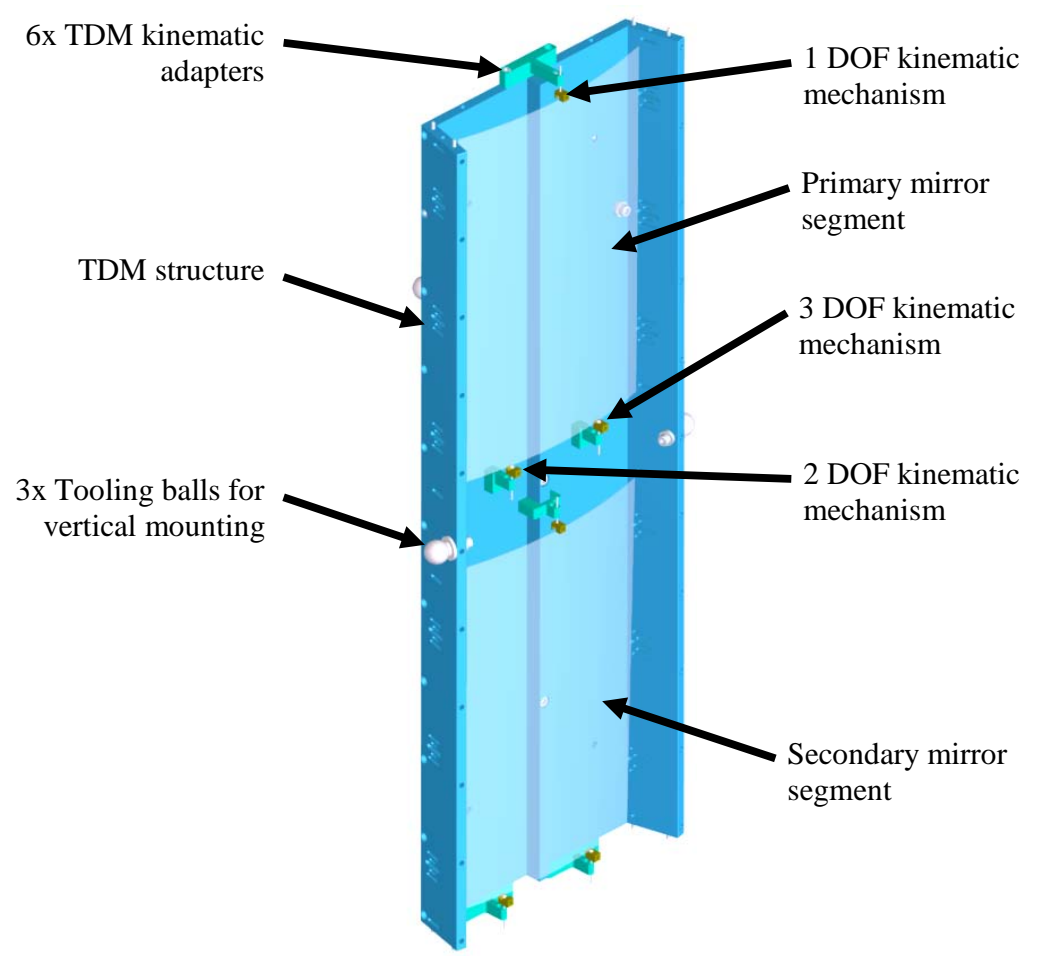

Figure 12. Modified TDM used to demonstrate kinematic mounting of a mirror pair.

\section{CONCLUSIONS}

As the $\sim 10$ arc-second slumped glass mirror technology being developed by the NGXO team nears readiness for mission implementation, extensive design, analysis, and testing has been conducted on Technology Demonstration Modules. These TDMs have been used to demonstrate multiple mirror pair co-alignment, X-ray performance, and compatibility with space-flight environments. Thermal vacuum and acoustic load tests have been performed and shown to not degrade optical performance. The most challenging environment has been random vibrations, where mirror response at high frequencies lead to large input vibration amplification and subsequent bond breakage or optical performance degradation. Strengthening the bonds through adhesive choice and increased bond area as well as reducing the amplification through vibration isolation have proven effective strategies at surviving increased random vibration input and are expected to allow the modules to meet random vibration load requirements in the near term.

The next generation of TDMs, which demonstrates a lightweight structure supporting more mirror segments, is currently being fabricated. Analysis predicts superior performance characteristics due to the use of E-60 Beryllium Metal Matrix Composite material with only a modest cost increase.

Preliminary steps are being taken to allow mounting and testing of 1-2 arc-second mirror segments expected to be available in the future. A Vertical X-ray Test Facility will minimize module gravity distortion and allow for less constrained mirror mounts, such as fully kinematic mounts. Permanent kinematic mounting into a modified TDM has been successfully demonstrated. 


\section{REFERENCES}

[1] Bookbinder, J. A., Smith R. K., Hornschemeier, A., et al, "The Constellation-X Observatory” Proc. of SPIE Vol. 7011, 701102, 2008.

[2] Zhang, W. W., et al., "Lightweight and high angular resolution x-ray optics for astronomy", Proc. SPIE 8076, 807602 (2011).

[3] Zhang, W. W., et al., "High resolution and high throughput x-ray optics for future astronomical missions", Proc. SPIE 8861-22, (2013).

[4] Zhang, W. W., et al., "Mirror technology development for the International X-ray Observatory mission (IXO)", Proc. SPIE 7732, 77321G (2010).

[5] McClelland, R. S., et al., "Design and analysis of the International X-Ray Observatory mirror modules ", Proc. SPIE 7732, (2010).

[6] McClelland, R. S., et al., "Design and Analysis of Modules for Segmented X-Ray Optics", Proc. SPIE 8443-144, (2012). 